\title{
Electromagnetism from 5D gravity: Beyond the Maxwell equations
}

\author{
Romulus Breban \\ Institut Pasteur, Paris, France
}

\begin{abstract}
Ever with the work of Kaluza, it has been known that 4D Einstein- and Maxwell-type equations can emerge from the field equations of 5D gravity in Ricci-flat space-times, having a space-like Killing vector. We revisit these equations and compare them with the Maxwell equations and the Ohm's law. Although 5D gravity and traditional electromagnetic theory are mathematically related, a paradigm shift in traditional electromagnetic concepts is required for unification. First, both the Maxwell equations and the Ohm's law are found among the field equations for 5D gravity, which gives Ohm's law the status of a field equation. Second, the concept of 4-current density, bringing together electrical charge and current density, is not fundamental for the electromagnetic theory resulting from 5D gravity. Third, the $5 \mathrm{D}$ gravity field equations suggest a generalization for the model of the perfect conductor, to describe a broader range of electromagnetic phenomena and bypass the concept of 4-current density. To see these ideas at work in a simple context, we propose an amendment to the Maxwell equations, based on the field equations of 5D gravity, and discuss a few applications. Namely, we discuss the propagation of electromagnetic waves through an imperfect conductor and topics in electrostatics and magnetostatics, touching ground with the Hall effect and the phenomenon of persistent current.
\end{abstract}

\section{Introduction}

The history of the electromagnetic theory is marked by philosophical choices, standardizations and leaps in understanding [1]. These may be revisited with progress in physics, to shed new light on the exceptional body of work in electromagnetism. The core equations for explaining the phenomenology are the Maxwell equations, written in space and time. The electromagnetic field is defined using three spatial coordinates, $x^{j}(j, k, l, \ldots=1,2,3)$, in 3D Euclidian geometry, while the time $t$ stands only as a parameter. However, the very structure of the Maxwell equations defines a $4 \mathrm{D}$ Lorentzian geometry, where time appears as a coordinate, $x^{0}$. In CGS units, the modern formulation of the Maxwell equations is (e.g., Refs. [2, Ch. 1] and $[3$, Ch. 6])

$$
\begin{aligned}
\nabla \boldsymbol{D} & =4 \pi \rho \\
\nabla \boldsymbol{B} & =0 \\
\nabla \times \boldsymbol{E} & =-\frac{1}{c} \frac{\partial \boldsymbol{B}}{\partial t} \\
\nabla \times \boldsymbol{H} & =\frac{1}{c} \frac{\partial \boldsymbol{D}}{\partial t}+\frac{4 \pi}{c} \boldsymbol{j}
\end{aligned}
$$

supplemented by constitutive/material equations

$$
\begin{aligned}
& \boldsymbol{D}=\boldsymbol{E}+4 \pi \boldsymbol{P} \\
& \boldsymbol{B}=\boldsymbol{H}+4 \pi \boldsymbol{M},
\end{aligned}
$$

(n.b., in vacuum, $\boldsymbol{P}=\boldsymbol{M}=0$ ) and Ohm's law

$$
\boldsymbol{j}=\varsigma \boldsymbol{E} .
$$

For mathematical convenience, the potentials $A_{0}$ and $\boldsymbol{A}$ are introduced for the electromagnetic field

$$
\begin{aligned}
\boldsymbol{E} & =-\nabla A_{0}-\frac{1}{c} \frac{\partial \boldsymbol{A}}{\partial t}, \\
\boldsymbol{B} & =\nabla \times \boldsymbol{A} .
\end{aligned}
$$


A recent critique of the Maxwell equations can be found in Ref. [4]. We make two additional notes. First, the Maxwell equations require the concepts of both field and source. Briefly, source generates field; the observer can act directly on the source, but not on the field. It is conjectured that electrical charge in motion is the source of the electromagnetic field. Second, as evidenced by Eqs. (1.5)-(1.7), the Maxwell equations make explicit assumptions about the structure of matter. In fact, many concepts such as conductor, dielectric, electron, were developed in parallel with the equations for the electromagnetic field, itself. Electromagnetic phenomena are attributed to particles, crystalline/amorphous structures and domain dynamics of matter. The relationship between electromagnetic phenomenology and the Maxwell equations remains complex; e.g., Ref. [5]. Here, our discussion is limited to fields, sources and particle electromagnetism in vacuum.

Features of the Maxwell equations can be recovered from a 5D geometry without compact dimensions. In 1921, Kaluza [6] proposed to unify the description of electromagnetic field in vacuum, with that of the $4 \mathrm{D}$ gravitational field, using a 5D space-time geometry where the metric is independent of the fifth dimension, $x^{5}$. Later, Wesson [7] elaborated the work of Kaluza, inspired by progress in 4D gravitation theory, particularly resisting Klein's idea [8] that the fifth dimension be compact. Specifically, Wesson [7] proposed an interpretation for the covariance breaking in the gravitational field variables, from five to four dimensions. We adopt the Kaluza-Wesson interpretation of induced matter, whereby sourceless 5D gravity can be regarded, in four dimensions, as electromagnetic field in vacuum, onto a space-time hosting $4 \mathrm{D}$ gravity. Thus, an empty (i.e., Ricci-flat) 5D space-time appears to contain active matter (i.e., sources of fields), when it is interpreted in four dimensions. Several authors published field equations resulting for 5D Ricci-flat space-times, which are compatible with the induced-matter interpretation [6,9-12]. However, covariance breaking of the 5D gravitational field, to obtain field equations of type Einstein and Maxwell, is not unique. There are many ways to parameterize the 5D metric and still obtain $4 \mathrm{D}$ field equations with desired properties. Here, we impose two requirements, which make the 5D field parameterization unique. First, the parameterization submits to the geometric picture of the foliation of the 5D metric along $x^{5}$ and, second, it provides an anomaly-free theory for single-particle propagation in $4 \mathrm{D}$ space-time, according to the principles of general relativity [13-16].

Geometrically speaking, there is nothing peculiar about the fifth dimension. Rather than using an $x^{5}$ independent 5D metric, we can use an $x^{0}$-independent 5D metric, foliate along $x^{0}$, and obtain Einsteinand Maxwell-type equations for 4D fields from the condition of 5D Ricci flatness; ${ }^{1}$ It is claimed that timeindependent particle propagation can be interpreted within a statistical physics formalism [13-15]. However, the Maxwell-type equations resulting in this case are associated with a 4D Euclidian metric rather than a 4D Lorentzian metric and it remains unclear how they link to the current phenomenology of electromagnetism. An alternative is to maintain the parameterization induced by the foliation along $x^{5}$, when discussing $x^{0}$ independent physics, as it guarantees an interpretation for the field variables [13-15]. These topics remain for further work.

The paper is structured as follows. In Sec. 2, we briefly review the theory for 5D null-propagation of passive particles; i.e., material particles which are not sources of fields. ${ }^{2}$ In Sec. 3, we explain how, in the case where the 5D metric is $x^{5}$-independent, $5 \mathrm{D}$ physics can undergo covariance breaking, to be interpreted as $4 \mathrm{D}$ mechanics. Indeed, the resulting $4 \mathrm{D}$ theory of passive particle propagation is anomaly free, in full agreement to the traditional equations of $4 \mathrm{D}$, classical and quantum, relativistic mechanics. The reader familiar with these developments may proceed directly to Sec. 4, where we discuss the field equations resulting in Ricci-flat 5D gravity, from the perspective of the Maxwell equations and Ohm's law. As with previous work [6,9-11], we make the case that 5D gravity is a successful physical theory, in touch with experiment. However, the traditional electromagnetic theory can gain, as well, from the parallel with field equations for 5D gravity. In particular, it can gain sharper concepts and, perhaps, a richer basis, as phenomenology finds reason to be included with the fundamentals. These aspects and others are discussed in Secs. 4.1 and 5. In Sec. 6, we conclude our work.

\footnotetext{
${ }^{1}$ This type of foliation is performed in the Hamiltonian formulation of $4 \mathrm{D}$ general relativity, for which the Arnowitt-DeserMisner foliation $[17,18]$ was originally intended.

${ }^{2}$ We do not consider the propagation of active massive (charged) particles propagating within the gravitational (electromagnetic) field that themselves produce.
} 


\section{$2 \quad 5 \mathrm{D}$ physics for $4 \mathrm{D}$ formalisms}

We postulate that reality is $5 \mathrm{D}$, yet an observer perceives/acknowledges only 4 dimensions out of 5 . For a complete physical picture or formalism, the observer relies on 4D geometry and an array of non-geometrical concepts. The physical pictures employed by a $4 \mathrm{D}$ observer result from different foliations of a $5 \mathrm{D}$ geometry [15]. It is postulated that, if the $5 \mathrm{D}$ geometry is independent of the fifth coordinate then the $5 \mathrm{D}$ physics appears as 4D mechanics, while if the 5D geometry is independent of time then the 5D physics appears as $4 \mathrm{D}$ statistical physics. Throughout the manuscript, we use bold face to indicate that some indices of the corresponding mathematical objects are suppressed, particularly for $3 \mathrm{D}$ vectors. We start with a $5 \mathrm{D}$ Lorentzian space-time, $\mathcal{M}^{(5)}$, with metric $h_{M N}(M, N, \cdots=0,1,2,3,5)$, having the signature $(-,+,+,+,+)$, that is sufficiently smooth and subject to the principle of general covariance. We further consider that $\mathcal{M}^{(5)}$ is Ricci flat; i.e., $R_{M N}^{(5)}=0$ and the $5 \mathrm{D}$ gravity fields have no sources. This is just a minimalist choice, not required by the induced matter principle.

We assume that the observed particle is passive, not a source of field interacting with the space-time metric. Particle propagation can be described using suitable equations defined on $\left(\mathcal{M}^{(5)}, \boldsymbol{h}\right)$. We are particularly interested in null propagation, therefore 5D optics, defined in two flavors. First, we can define classical or geometrical optics, based on 5D null geodesics. Second, we can define quantum optics. A spinless formulation is provided by the $5 \mathrm{D}$ wave equation, where the wavefunction $\Psi(\cdot, \cdot)$ may be regarded as the number of null paths between any two given points of $\mathcal{M}^{(5)}$. A spin-1/2 quantum optics requires a wavefunction $\boldsymbol{\Psi}$, which is a pair of bispinors or a biquaternion [13], a vector with two quaternionic components, at every point of $\mathcal{M}^{(5)}$. It can be argued that $\boldsymbol{\Psi}$ represents two tangent null vectors, one incoming and one outgoing, at every point of $\mathcal{M}^{(5)}$ [13]. The wavefunction equation is of Dirac-type, based on a fünfbein field ${ }^{3}$ and a special Clifford algebra; see Eq. (14) in Ref. [13]. The focus is, thus, solving null-propagation problems for passive particles, given the $5 \mathrm{D}$ gravity field. ${ }^{4}$ The propagation problems are invariant to conformal transformations of $h_{M N}$, which cannot be fully recovered by observing only particles in null propagation. We add tilde to the metric symbol, $\tilde{h}_{M N}$, to denote a conformally transformed metric $h_{M N}$.

Among space-times suitable for applications are 5D black holes, where the null structure has been a topic of major interest [19-22]. It is interesting to note that, while the event horizon of a 4D black hole is always of spherical topology, the event horizon of a $5 \mathrm{D}$ black hole can have spherical, toroidal, or other topologies [22]. Here, we propose 4D interpretations of 5D space-times and passive-particle null-propagation in $5 \mathrm{D}$ space-times $[13-15]$.

\section{4D classical and quantum mechanics from 5D physics}

Interpretation of the 5D geometry and dimensional reduction are greatly facilitated by symmetry. The isometries of $\left(\mathcal{M}^{(5)}, \boldsymbol{h}\right)$ form a Lie group, denoted $\operatorname{Iso}\left(\mathcal{M}^{(5)}, \boldsymbol{h}\right)$. The generators of this group are called Killing vectors, $\boldsymbol{\xi}$, satisfing the Killing equation $\partial_{M} \xi_{N}+\partial_{N} \xi_{M}=0$. We select a nontrivial subgroup of Iso $\left(\mathcal{M}^{(5)}, \boldsymbol{h}\right)$, which we call the formalism isometry group, FIso $\left(\mathcal{M}^{(5)}, \boldsymbol{h}\right)$, and its elements, formalism isometries. Here, we consider that $\left(\mathcal{M}^{(5)}, \boldsymbol{h}\right)$ has only one formalism isometry: the translation along a space-like dimension, $x^{5} .{ }^{5}$ We foliate $\left(\mathcal{M}^{(5)}, \boldsymbol{h}\right)$ along the formalism Killing field, into 4D space-times, using the Arnowitt-Deser-Misner procedure [17,18], and indicate the lapse and shift fields of the foliation in the 5D metric. In turn, this yields a geometrical parameterization of $h_{M N}$, which we further write in adapted coordinates, so it does not depend on $x^{5}$. We thus obtain

$$
h^{M N}=\left(\begin{array}{cc}
g^{\mu \nu} & N^{\mu} \\
N^{\nu} & N^{\rho} N_{\rho}+N^{2}
\end{array}\right) \Leftrightarrow h_{M N}=\left(\begin{array}{cc}
g_{\mu \nu}+N_{\mu} N_{\nu} / N^{2} & -N_{\mu} / N^{2} \\
-N_{\nu} / N^{2} & 1 / N^{2}
\end{array}\right),
$$

\footnotetext{
${ }^{3}$ The fünfbein field specifies a choice for a local frame at each point of the $5 \mathrm{D}$ manifold.

${ }^{4}$ The $5 \mathrm{D}$ observer has no notion of particle charge or mass and does not distinguish between electric and gravitational fields.

${ }^{5}$ Mathematics allows for much more general theories. For example, we may consider a space-time with a time-like Killing vector or several Killing vectors as formalism isometries, possibly requiring foliation over several dimensions of the 5D manifold. The difficulty resides in the physical interpretation and link to experiment.
} 
where $\left(\mathcal{M}^{(4)}, \boldsymbol{g}\right)$ is the foliated $4 \mathrm{D}$ manifold and, $N$ and $N_{\mu}(\mu, \nu, \ldots=0,1,2,3)$, respectively, are the lapse and shift of the $4 \mathrm{D}$ foliation. We introduce physical notation

$$
N_{\mu} \equiv-\frac{q}{c^{2}} A_{\mu}, \quad N \equiv \Phi^{-1},
$$

where $A_{\mu}$ represents the electromagnetic potential in CGS units; $q$ is specific charge and $c$ is the speed of light in vacuum. As it turns out, the $4 \mathrm{D}$ metric, which yields the well-known laws of $4 \mathrm{D}$ particle propagation, is not $g_{\mu \nu}$, but $\tilde{g}_{\mu \nu} \equiv g_{\mu \nu} / \Phi^{2}$ [15]. The 5D metric observed through particle propagation in 4D mechanics is $\tilde{h}_{M N}=h_{M N} / \Phi^{2}$; this gives the second requirement for the parameterization of the $5 \mathrm{D}$ metric. We write the metric $h_{M N}$ using the fields that are observed in particle propagation; see Sec. 4.

The 4D observer may perform any cylindrical transformation of coordinates, consisting of diffeomorphisms of $\left(\mathcal{M}^{(4)}, \tilde{\boldsymbol{g}}\right)$ leaving $x^{5}$ unchanged; i.e.,

$$
x^{\mu} \rightarrow \mathcal{F}^{\mu}\left(x^{\nu}\right), \quad x^{5} \rightarrow x^{5} .
$$

Other coordinate transformations, not changing 4D physical observables, are gauge transformations of the shift of the foliation, $N_{\mu}$, or the electromagnetic potential, $A_{\mu}$

$$
\left\{\begin{array} { l } 
{ x ^ { \mu } \rightarrow x ^ { \mu } , } \\
{ x ^ { 5 } \rightarrow x ^ { 5 } - \frac { q } { c ^ { 2 } } \mathcal { A } ( x ^ { \mu } ) , }
\end{array} \Leftrightarrow \left\{\begin{array}{l}
\tilde{g}_{\mu \nu} \rightarrow \tilde{g}_{\mu \nu}, \\
A_{\mu} \rightarrow A_{\mu}+\partial_{\mu} \mathcal{A}\left(x^{\nu}\right), \\
\Phi \rightarrow \Phi,
\end{array}\right.\right.
$$

where $\mathcal{A}\left(x^{\nu}\right)$ is the gauge function for $A_{\mu}$. It is unclear how the $4 \mathrm{D}$ observer would use other coordinate transformations and what is their physical interpretation within $4 \mathrm{D}$ mechanics.

The symmetry of $\left(\mathcal{M}^{(5)}, \boldsymbol{h}\right)$ to translation along $x^{5}$ is key for describing 5D passive particles of null mass (i.e., 5D photons), in four dimensions, as 4D passive particles carrying mass and electrical charge. The 4D observer considers himself/herself uncharged, which leads to important choices in the process of dimensional reduction. For classical propagation, 5D null geodesics in $\left(\mathcal{M}^{(5)}, \boldsymbol{h}\right)$ are given $4 \mathrm{D}$ interpretation [15]. If the uncharged 4D observer adopts the path element of $\left(\mathcal{M}^{(4)}, \tilde{\boldsymbol{g}}\right)$ for the infinitesimal proper time, then 5D null geodesics are represented as $4 \mathrm{D}$ time-like Randers geodesics $[23,24]$ in $\left(\mathcal{M}^{(4)}, \tilde{\boldsymbol{g}}\right)$, where the eccentricity of the path element is determined by $A_{\mu}$. Furthermore, since the $x^{5}$-translational symmetry yields a conserved quantity for $5 \mathrm{D}$ geodesic motion, we say that the $4 \mathrm{D}$ particle propagates with constant passive mass [15]. For quantum propagation, we perform Fourier transforms with respect with $x^{5}$ of the $5 \mathrm{D}$ wave equation and the 5D Dirac-type equation, which, in addition, requires a foliated fünfbein field [13]. ${ }^{6}$ It is further postulated that the coordinate $x^{5}$ is conjugated with the inverse Compton wavelength $\lambda^{-1}=m c / \hbar[13,14]$. In this fashion, 5D photons are interpreted as 4D on-shell massive quantum particles.

A priori, there is no reason for the $5 \mathrm{D}$ space-time be symmetric, in which case, we do not offer a physical picture for the 5D geometry. However, if the 5D space-time is nearly symmetric, then a interpretation can be constructed based on the alleged symmetry and a top-down hierarchy of physical concepts. The first order concepts are those of the physical picture assuming that the symmetry is exact. They provide a satisfactory description for particle propagation in manifold fields, for a small enough proper-time interval and space-time neighborhood. Next to the first order physical concepts, second order concepts can be added for improving the description of particle propagation, so it holds for longer proper-time intervals and larger space-time neighborhoods. In general, the physical concepts of second order rely on those of first order. Therefore, the hierarchical physical interpretation is not covariant. Reference [16] demonstrates how particle propagation in a 5D space-time with approximate $x^{5}$-translation symmetry can be given a physical picture. In the first order, we use 4D mechanics [15]. For the second order, we invoke additional phenomena such as Stokes friction and non-hermitian quantum decoherence [16].

\section{Field equations in 4D mechanics}

Several authors [9-12] published the field equations for $x^{5}$-independent $5 \mathrm{D}$ gravity, $R_{M N}^{(5)}=0$, in the parametrization given by $g_{\mu \nu}, A_{\mu}$ and $\Phi$. We performed an additional conformal transformation and rewrote

\footnotetext{
${ }^{6}$ The choice for the foliated fünfbein rests with the uncharged $4 \mathrm{D}$ observer [14].
} 
the equations for $5 \mathrm{D}$ gravity in the fields $\tilde{g}_{\mu \nu}, A_{\mu}$ and $\Phi$, which have more physical content because $\tilde{g}_{\mu \nu}$ is the $4 \mathrm{D}$ metric observed for $4 \mathrm{D}$ passive particle propagation [15]

$$
\begin{aligned}
\tilde{R}_{\mu \nu}^{(4)}-\frac{1}{2} \tilde{g}_{\mu \nu} \tilde{R}^{(4)} & =\frac{q^{2}}{2 c^{4}} \tilde{T}_{\mu \nu}^{E M}+\tilde{T}_{\mu \nu}^{\Phi}, \\
\tilde{\nabla}^{\mu} \tilde{F}_{\mu \nu} & =-3 \tilde{F}_{\mu \nu} \tilde{\nabla}^{\mu} \ln \Phi, \\
\frac{1}{\Phi} \tilde{\nabla}^{\mu} \tilde{\nabla}_{\mu} \Phi & =\frac{q^{2}}{4 c^{4}} \tilde{F}_{\mu \nu} \tilde{F}^{\mu \nu}+\frac{1}{6} \tilde{T}_{\mu \nu}^{\Phi} \tilde{g}^{\mu \nu},
\end{aligned}
$$

where $\tilde{R}_{\mu \nu}^{(4)}$ and $\tilde{\nabla}_{\mu}$ are, respectively, the $4 \mathrm{D}$ Ricci tensor and the Levi-Civita connection of the metric $\tilde{g}_{\mu \nu}$. Furthermore, $\tilde{F}_{\mu \nu} \equiv F_{\mu \nu}=\partial_{\mu} A_{\nu}-\partial_{\nu} A_{\mu}$ and

$$
\begin{aligned}
\tilde{T}_{\mu \nu}^{E M} & =\tilde{g}_{\nu \rho} \tilde{F}_{\mu \sigma} \tilde{F}^{\sigma \rho}+\frac{1}{4} \tilde{g}_{\mu \nu} \tilde{F}_{\rho \sigma} \tilde{F}^{\rho \sigma}, \\
\tilde{T}_{\mu \nu}^{\Phi} & =\frac{1}{\Phi}\left[\tilde{\nabla}_{\mu} \tilde{\nabla}_{\nu} \Phi-\frac{2}{\Phi}\left(\tilde{\nabla}_{\mu} \Phi\right)\left(\tilde{\nabla}_{\nu} \Phi\right)\right]-\frac{1}{\Phi} \tilde{g}_{\mu \nu} \tilde{g}^{\rho \sigma}\left[\tilde{\nabla}_{\rho} \tilde{\nabla}_{\sigma} \Phi-\frac{2}{\Phi}\left(\tilde{\nabla}_{\rho} \Phi\right)\left(\tilde{\nabla}_{\sigma} \Phi\right)\right],
\end{aligned}
$$

are the stress-energy tensors for the electromagnetic and $\Phi$ fields, respectively. These equations are interpreted according to the induced matter principle. An empty 5D space-time, equipped with a space-like Killing field, is regarded as a $4 \mathrm{D}$ space-time containing electromagnetic and $\Phi$ fields, as sources of $4 \mathrm{D}$ gravity. Equation (4.12) represents the 4D Einstein equations where the dichotomy between fields and sources occurs only with the $4 \mathrm{D}$ induced-matter interpretation of the $5 \mathrm{D}$ geometry. The RHS of Eq. (4.12) is separated into electromagnetic and $\Phi$ sources. It is important to note that the $4 \mathrm{D}$ gravity sources are different in nature: $\tilde{T}_{\mu \nu}^{E M}$ can be inferred from passive particle propagation, while $\tilde{T}_{\mu \nu}^{\Phi}$ cannot. Therefore, from this perspective, $\Phi$ appears like a dark field $[25,26]$.

\subsection{The Maxwell equations and Ohm's law from the equations for 5D gravity}

Equation (4.13) is of type Maxwell and helps recover Eqs. (1.1) and (1.4), considered in vacuum. ${ }^{7}$ For this, we break Eq. (4.13) to introduce the 4-current density, $j_{\nu}$

$$
\begin{aligned}
\tilde{\nabla}^{\mu} \tilde{F}_{\mu \nu} & \equiv \frac{4 \pi}{c} j_{\nu}, \\
\frac{4 \pi}{c} j_{\nu} & \equiv-3 \tilde{F}_{\mu \nu} \tilde{\nabla}^{\mu} \ln \Phi .
\end{aligned}
$$

Eq. (4.17) corresponds to the Maxwell equations (1.1) and (1.4); the RHS of Eq. (4.17) expresses the sources for the electromagnetic field in the LHS. Equation (4.18) further explicates $j_{\mu}$ in terms of $\Phi$ and the electromagnetic field, and is interpreted in the same way: the RHS designates the sources for the LHS. The alternative interpretation, where the LHS designates the source for the electromagnetic and $\Phi$ fields in the RHS, yields a contender to the Maxwell equations, and a line of argument far from the traditional electromagnetic theory. We will not pursue it here. Applying $\tilde{\nabla}^{\nu}$ on both sides of Eq. (4.18) and using Eq. (4.13) yields the $4 \mathrm{D}$ continuity equation for $j_{\nu}$ as an identity ${ }^{8}$

$$
\frac{4 \pi}{c} \tilde{\nabla}^{\nu} j_{\nu}=-3\left(\tilde{\nabla}^{\nu} \tilde{F}_{\mu \nu}\right)\left(\tilde{\nabla}^{\mu} \ln \Phi\right)-3 \tilde{F}_{\mu \nu} \tilde{\nabla}^{\nu} \tilde{\nabla}^{\mu} \ln \Phi=6 \tilde{F}_{\mu \nu} \tilde{\nabla}^{\nu} \tilde{\nabla}^{\mu} \ln \Phi=0
$$

It is significant that $j_{\nu}$ depends on $\Phi$, which does not appear in the LHS of Eq. (4.17). Still, as a divergencefree vector field, $j_{\nu}$ retains more freedom than $\Phi$. From the perspective of a $4 \mathrm{D}$ field theory, $j_{\nu}$ requires no

\footnotetext{
${ }^{7}$ The concept of vacuum is vague in terms of Lorentzian geometry. According to the induced matter principle, $F_{\mu \nu}$ is an active matter field defined on $4 \mathrm{D}$ space-time. However, the reference, empty $4 \mathrm{D}$ space-time is Ricci flat, with field equations $R_{M N}^{(4)}=0$, where $F_{\mu \nu}$ and $\Phi$ do not not exist. Therefore, the reference $4 \mathrm{D}$ space-time is not sufficient for describing particle propagation in electromagnetic field, in vacuum. We are lead to postulate that vacuum represents $5 \mathrm{D}$ emptiness rather than $4 \mathrm{D}$ emptiness; it is unclear how this relates to experiment. Another possibility is to associate vacuum to the limit of weak fields evolving in a $4 \mathrm{D}$ space-time. The term electrovacuum is often used for solutions of both Einstein and Maxwell equations.

${ }^{8}$ This is also obtained by applying $\tilde{\nabla}^{\nu}$ on both sides of Eq. (4.17).
} 
further analysis. It is very important to note that, in the theory of $5 \mathrm{D}$ gravity, $j_{\nu}$ results from a dispensable ansatz (i.e., Eqs. (4.17) and (4.18)), of no particular benefit for solving the 5D field equations. This is in stark contrast with the fundamental role that $j_{\nu}$ plays in the theories of electromagnetism and condensed matter, where a fundamental SI unit is dedicated to measurements of electrical current. These theories further describe $j_{\nu}$, postulating conduction mechanisms which rely on the structure of matter. For example, in solid state physics (e.g., Refs. [27, Chs. 1, 2] and [28, Ch. 6]), the current density is described using classical or quantum formalisms for passive particles/quasiparticles in motion, trapped within matter, subject to the Lorentz force. In addition, the particles/quasiparticles are subject to collisions or phenomenological friction forces, due to additional electromagnetic interactions associated to the atomic structure of matter. ${ }^{9}$ In plasma physics (e.g., Refs. [29, Ch. 1] and [30, Chs. 5, 6]), conduction mechanisms have similar building principles, although, sometimes, they simply postulate that the Lorentz force is zero for particles within plasma. A 3D velocity field for charged carriers is often defined using $4 \mathrm{D}$ active matter $\boldsymbol{v} / c \equiv \boldsymbol{j} / j^{0} .{ }^{10}$

There are two main uses for the microscopic pictures of $j_{\nu}$. First, they explain the Laplace force [31], the force acting on a conductor placed in electromagnetic field, in terms of the Lorentz force, ${ }^{11}$ see e.g., [3, Ch. 5.2] for an argument based on Ampére's work [32]. However, the Laplace force does not require an explanation via the Lorentz force, because the Laplace force can be obtained from the divergence of the electromagnetic stress-energy tensor, $\tilde{\nabla}^{\mu} \tilde{T}_{\mu \nu}^{E M}$ [3, Ch. 11.11]. Second, they provide microscopic explanations for the Ohm's law and other laws in condensed matter physics. However, as we explain below, a conduction mechanism is not required to explain Ohm's law, which here appears as a field equation. To see this, we provide a physical interpretation for Eq. (4.18), breaking 4D covariance in the limit of weak fields.

\section{A minimal amendment to the Maxwell equations}

The rigorous approach to Eqs. (4.12)-(4.14) is through exact solutions, analyzed for their relevance to experiment. Many exact solutions for empty 5D space-times without compact dimensions have already been found $[20,33-39]$, but they primarily describe time-independent geometries, which are not invariant to translation along $x^{5}$, and do not satisfy Eqs. (4.12)-(4.14). Perhaps, the only exception is a class of time-dependent solutions, obtained through the Wick rotation of the 5D Myers-Perry black-hole metric $[12,40,41]{ }^{12}$

An alternate approach is using the 5D field equations for an amendment to the Maxwell equations, which are already close to experiment. Evidently, the physics of the amended Maxwell equations is superseded by that of corresponding exact 5D solutions. We propose a strong approximation of the 5D gravity equations (4.12)-(4.14) in the limit of weak fields. We consider that the $4 \mathrm{D}$ metric, $\tilde{g}_{\mu \nu}$, is approximately the Minkowski metric, $\eta_{\mu \nu}$; n.b., $\left(q / c^{2}\right)^{2} \tilde{\boldsymbol{T}}^{E M} / 2+\tilde{\boldsymbol{T}}^{\Phi} \approx 0$. We further break covariance to distinguish 3D space and time, and write the $4 \mathrm{D}$ connection as $\tilde{\nabla}^{\nu} \equiv\left(\tilde{\partial}^{0}, \nabla\right)$. In (pseudo)cartesian coordinates, the 3D space metric is $\operatorname{diag}(1,1,1)$, so we no longer distinguish covariant and contravariant spatial components of tensors. Furthermore, we use the Levi-Civita symbol, $\epsilon_{j k l}$, as a cartesian tensor, and introduce the 3D curl operator and vector product. Setting $x^{0} \equiv-c t$, and using Eqs. (1.8) and (1.9), we introduce the 3D vector field variables

$$
\boldsymbol{E}=\tilde{F}_{0 j}, \quad B_{j}=\epsilon_{j k l} \tilde{F}_{k l} / 2 .
$$

Next to Eqs. (1.2) and (1.3), Eq. (4.13) yields

$$
\begin{aligned}
\nabla \boldsymbol{E} & =\frac{4 \pi}{c} \boldsymbol{\varrho} \boldsymbol{E}, \\
\nabla \boldsymbol{B} & =0 \\
\nabla \times \boldsymbol{E} & =-\frac{1}{c} \frac{\partial \boldsymbol{B}}{\partial t}, \\
\nabla \times \boldsymbol{B} & =\frac{1}{c} \frac{\partial \boldsymbol{E}}{\partial t}+\frac{4 \pi}{c}(\varsigma \boldsymbol{E}+\varrho \times \boldsymbol{B}),
\end{aligned}
$$

\footnotetext{
${ }^{9}$ These atomic electromagnetic fields are not included in the Maxwell equations with the sources $j_{\nu}$.

${ }^{10}$ Our theory does not require $4 \mathrm{D}$ active matter satisfy the $4 \mathrm{D}$ on-shell constraint, designed for $4 \mathrm{D}$ passive particles.

${ }^{11}$ The Lorentz force acts on passive particles.

${ }^{12}$ Identifying $z$ with $x^{5}$ in Eq. (1) of Ref. [12] shows that the 5D metric has nontrivial fields $\tilde{g}_{\mu \nu}, A_{\mu}$ and $\Phi$. The induced matter interpretation of 5D metric remains for further work.
} 
where the fields

$$
\varrho \equiv-\frac{c}{4 \pi} \nabla \ln \Phi^{3}, \quad \varsigma \equiv \frac{1}{4 \pi} \frac{\partial \ln \Phi^{3}}{\partial t},
$$

are regarded to be material properties, satisfying

$$
-\frac{1}{c} \frac{\partial \varrho}{\partial t}=\nabla \varsigma
$$

Alternatively, one can directly choose a model for the field $\Phi .{ }^{13}$ Electrical charge and current densities are not needed for the amended Maxwell equations (5.21)-(5.24), but they result from Eq. (4.18) as

$$
\begin{aligned}
\rho & =\varrho \boldsymbol{E} / c, \\
\boldsymbol{j} & =\varsigma \boldsymbol{E}+\boldsymbol{\varrho} \times \boldsymbol{B} .
\end{aligned}
$$

Equations (5.27) and (5.28) can be regarded as a generalization of the Ohm's law. Conceptually, a perfect conductor has very high conductivity, does not hold charge density in volume, nor allows for magnetic field inside the material [3, Ch. 8.1]. This model is recovered in the above equations by assuming that $\Phi$ is approximately independent of space (i.e., $\nabla \ln \Phi^{3} \approx 0$ or $\varrho \approx 0$ ) in some region, where we say the perfect conductor is found. According to Eq. (5.27), the charge density is approximately zero, and Eq. (5.28) yields the Ohm's law (1.7). Equations (5.27) and (5.28) thus describe a more realistic, imperfect conductor, which holds electric charge in volume and allows penetration by the magnetic field. Hence, the conduction current $\boldsymbol{j}$ has an ohmic component and a non-ohmic component, which depends on the local magnetic field. These phenomena are characterized by the $3 \mathrm{D}$ vector field $\varrho$, which is regarded here as a material property, next to the conductivity $\varsigma$.

Taking an imperfect conductor for being perfect can have important consequences for the interpretation of experimental data. The ordinary Hall effect $[27,28,42]$ clearly demonstrates that an external magnetic field penetrates conductor materials, in contradiction to the model for the perfect conductor. ${ }^{14}$ Still, if the model of a perfect conductor is used to interpret experimental data, then all conduction current is considered to be ohmic and, apparently, the conductivity depends linearly on the local magnetic field; c.f., Eqs. (5.28). Furthermore, if the external magnetic field is large compared to the magnetic field generated by the electric current $\boldsymbol{j}$, then it appears that the conductivity depends linearly on the external magnetic field. These considerations seem to explain experimental observations by Kapitza [43-45], regarding a large number of chemical elements. It may however be possible to reinterpret Kapitza's experimental data using the model of an imperfect conductor (c.f., Eq. (5.27) and (5.28)), with the benefit of a simpler picture for the material properties. The phenomena by which an applied magnetic field changes the conductivity ${ }^{15}$ of a material are known under the generic name of magnetoresistance [27,28]. This is a large and complex chapter in condensed matter physics. The imperfect conductor model may help disentangle this complexity, while making no assumptions about the structure of matter, only using 5D field theory.

Another example of a phenomenon where the concept of imperfect conductor may be important is that of persistent current [46-48], which was discovered in gold and other metals. Consider an imperfect conductor in zero electric field and nonzero external magnetic field; this may be, for example, the magnetic field of a probe. According to Eq. (5.28), the external magnetic field causes a current density $\varrho \times \boldsymbol{B}$ inside the conductor, where $\boldsymbol{B}$ is the local magnetic field inside the conductor. In turn, this current density causes an additional component to the external magnetic, as described by the Maxwell equations. In the model of the perfect conductor, this looks like an instantaneous field response, otherwise, one is lead to assume a strange ohmic current inside the perfect conductor, even in the absence of electric field. We hope that the model of an imperfect conductor presented here can help provide a simple and natural explanation for the phenomenon of persistent current, within the framework of classical field theory.

\footnotetext{
${ }^{13}$ The weak field limit of Eq. (4.14) may provide an equation for $\Phi$. Here, we adopt a more general view, where the choice of $\Phi$ is only limited by its success in applying Eqs. (5.21)-(5.24) to experiment.

${ }^{14}$ Hall discovered the effect in gold, which does not behave like a perfect conductor. This raises questions about the applicability of the model of the perfect conductor.

${ }^{15}$ The conductivity remains defined by the Ohm's law, within the perfect conductor model.
} 
Lastly, we briefly comment on the Hall effect $[27,28,42]$. Using the perfect conductor model, the effect can be described that applying magnetic field transverse to ohmic current flow triggers an additional current component, transverse to both the initial ohmic current and the applied magnetic field. The traditional explanation of the Hall effect is based on conduction mechanisms, violating both the perfect and imperfect conductor models. It is assumed that the external magnetic field penetrates the conductor material and changes the motion of the conducting particles inside. Consequently, the conducting particles, considered as being passive, deviate from their initial trajectories, creating an additional current component inside the conductor. ${ }^{16}$ However, within an imperfect conductor, even without applying a magnetic field, the conducting particles form a current, and already propagate within the magnetic field which they, themselves, create (Ampère's law). Therefore, the conducting particles within an imperfect conductor constitute active matter and cannot be described as passive particles. An alternate explanation of the Hall effect, free of conduction mechanisms, based only on the classical field theory and the concept of the imperfect conductor, would not have these difficulties. In fact, the phenomenon of persistent current and the Hall effect are very much related, both regarding penetration of magnetic field inside an imperfect conductor. The difference is that, for the Hall effect, the conductor is subject to ohmic current, next to the external magnetic field. In the remaining of this section, we discuss several applications of the amended Maxwell equations (5.21)-(5.24) to traditional problems formulated for the Maxwell equations.

\subsection{On the propagation of electromagnetic waves within a conducting material}

The most natural application of Eqs. (5.21)-(5.24) may be to describing the propagation of electromagnetic waves through conducting materials. Traditionally, the problem is formulated by assuming a perfect conductor and merging the Maxwell equations with the Ohm's law. This results in Eqs. (5.21)-(5.24), where $\varsigma$ is a constant and $\varrho$ is zero; see e.g., Refs. [3, Ch. 7.7], [49, Ch. 9.4] and [50, Ch. 13]. We slightly generalize this application to the case where both $\varsigma$ and $\varrho$ are constants. ${ }^{17}$ We proceed by deriving an equation for the electric field, $\boldsymbol{E} .{ }^{18}$ First, we take a curl on both sides of Eq. (5.23) and use Eq. (5.24) to substitute $\nabla \times \boldsymbol{B}$

$$
\nabla(\nabla \boldsymbol{E})-\nabla^{2} \boldsymbol{E}=-\frac{1}{c} \frac{\partial}{\partial t}\left(\frac{1}{c} \frac{\partial \boldsymbol{E}}{\partial t}+\frac{4 \pi}{c} \varsigma \boldsymbol{E}+\frac{4 \pi}{c} \boldsymbol{\varrho} \times \boldsymbol{B}\right) .
$$

Second, in Eq. (5.29), we substitute $\nabla \boldsymbol{E}$ using Eq. (5.21) and $\partial \boldsymbol{B} / \partial t$ using Eq. (5.23)

$$
\frac{4 \pi}{c} \nabla(\boldsymbol{E} \varrho)=\nabla^{2} \boldsymbol{E}-\frac{1}{c^{2}} \frac{\partial^{2} \boldsymbol{E}}{\partial t^{2}}-\frac{4 \pi \varsigma}{c^{2}} \frac{\partial \boldsymbol{E}}{\partial t}+\frac{4 \pi}{c} \varrho \times(\nabla \times \boldsymbol{E}) .
$$

We now search for plane wave solutions of type

$$
\boldsymbol{E}=\boldsymbol{E}_{0} \exp (i \boldsymbol{k} \boldsymbol{x}-i \omega t)
$$

where $i$ is the complex unit, $\boldsymbol{E}_{0}$ and $\omega$ are real constants, but we allow $\boldsymbol{k}$ to be a complex constant. We thus obtain the following dispersion equation in the propagation vector $\boldsymbol{k}$

$$
\frac{\omega^{2}}{c^{2}}-\boldsymbol{k}^{2}+\frac{4 \pi i}{c}\left(\frac{\varsigma \omega}{c}-\varrho k\right)=0
$$

In the case where $\varrho=0$, we simply recover the formula for a perfect conductor [3, Ch. 7.7]. The propagation vector $\boldsymbol{k}$ is complex, indicating that the electromagnetic wave can propagate within a perfect conductor, but it is attenuated over the length scale $1 / \operatorname{Im}|\boldsymbol{k}| \propto \varsigma^{-1 / 2}$, called skin or penetration depth. Equation (5.32) also shows that an imperfect conductor behaves like a perfect conductor, provided $\varrho \boldsymbol{k}=0$. This represents an anisotropy in the electromagnetic wave propagation. Notably, the anisotropy does not occur in the model of a perfect conductor and can help measure $\varrho$, assessing how imperfect the conductor material is. Wave propagation through an imperfect conductor is intrinsically anisotropic, where the anisotropy relates to $\varrho$, or

\footnotetext{
${ }^{16}$ It is intriguing that magnetoresistance never enters the discussion about the Hall effect. According to the experiments of Kapitza [43-45], magnetoresistance always occurs next to the Hall effect.

${ }^{17}$ It remains unclear how applicable to experiment is this choice for $\varsigma$ and $\varrho$.

${ }^{18}$ The same results are obtained by analyzing the equations for the magnetic field.
} 
the dark field $\Phi$. This is different from other sources of anisotropy, generally associated to the tensor nature of the relative electric permittivity or magnetic permeability, which here are taken for unit scalars. It is remarkable that, according to Eq. (5.32), electromagnetic waves can propagate loss-free within an imperfect conductor given that

$$
|\boldsymbol{k}|=\omega / c, \quad \cos \theta=\varsigma /|\varrho|
$$

where $\theta$ is the angle between $\varrho$ and $\boldsymbol{k}$, which has no imaginary part. The propagating wave solution requires $\varsigma \leq|\varrho|$, which cannot occur for a perfect conductor. Finally, we note that recent studies of electromagnetic wave propagations [51-56] take precautions to model realistic features of matter, yet do not go beyond the concept of perfect conductor.

\subsection{On electrostatics and magnetostatics}

We submit that Eqs. (5.21)-(5.24) can describe the electromagnetic field in a large number of applications, not just wave propagation. To discuss electrostatic and magnetostatic solutions, we consider a 5D metric which is independent of both $x^{5}$ and $x^{0}$. Therefore, $\Phi$ is time-independent, the conductivity $\varsigma$ is zero, and the ohmic component of the electric current density is zero, as well. Consequently, the framework cannot address the traditional magnetostatic theory [3,32], unless the ohmic component of the electric current density is zero. This is too restrictive to recover the law of Biot and Savart, or that of Ampère. The field equations become

$$
\begin{aligned}
\nabla \boldsymbol{E} & =\frac{4 \pi}{c} \boldsymbol{\varrho} \boldsymbol{E}, \\
\nabla \boldsymbol{B} & =0 \\
\nabla \times \boldsymbol{E} & =0 \\
\nabla \times \boldsymbol{B} & =\frac{4 \pi}{c} \boldsymbol{\varrho} \times \boldsymbol{B},
\end{aligned}
$$

where, based on the definitions $(5.25), \varrho \equiv-(c / 4 \pi) \nabla \ln \Phi^{3}$ has zero curl; i.e., $\nabla \times \varrho=0$. The electrostatic and magnetostatic sources depend on the very fields they determine

$$
\begin{aligned}
& \rho=\varrho \boldsymbol{E} / c, \\
& \boldsymbol{j}=\boldsymbol{\varrho} \times \boldsymbol{B} .
\end{aligned}
$$

However, these concepts are not strictly needed for the field equations. The equations for the electric and magnetic fields are determined by the same vector field, ${ }^{19} \boldsymbol{\varrho}$, yet they are decoupled. Either $\boldsymbol{E}$ or $\boldsymbol{B}$ can be zero, irrespective of the other. There are, of course, solutions where both $\boldsymbol{E}$ and $\boldsymbol{B}$ are nonzero, determined by $\varrho$. The following results through straightforward calculations.

Proposition. Under the change of variables

$$
\boldsymbol{E} \rightarrow \boldsymbol{E}^{*} \Phi^{-3}, \quad \boldsymbol{B} \rightarrow \boldsymbol{B}^{*} \Phi^{-3}
$$

Eqs. (5.34)-(5.37) remain invariant, where

$$
(\boldsymbol{E}, \boldsymbol{B}, \varrho) \rightarrow\left(\boldsymbol{B}^{*}, \boldsymbol{E}^{*},-\varrho\right)
$$

In consequence, it is sufficient to solve the equations for either electrostatics (i.e., Eqs. (5.34) and (5.36)) or magnetostatics (i.e., Eqs. (5.35) and (5.37)) for a complete solution. For example, the electrostatic equations can be combined into a single one, for the electrostatic potential,

$$
\nabla^{2} A_{0}=\frac{4 \pi}{c} \varrho \nabla A_{0}
$$

\footnotetext{
${ }^{19}$ Taking $\varrho=0$ accommodates all field configurations where $\boldsymbol{E}$ and $\boldsymbol{B}$ are independent constant vectors.
} 
that can be used to formulate homogeneous problems of type Dirichlet and Neumann; see Appendix A for alternate formulations of the Eq. (5.42). The solutions for $A_{0}$ generate all solutions of Eqs. (5.34)-(5.37).

The typical problem in electrostatics and magnetostatics is finding field configurations due to localized sources; it is assumed that the resulting field is also localized, thus vanishes at infinity. We discuss two elementary solutions for Eqs. (5.34)-(5.37). If all the vector fields are spherically symmetric, we obtain $\boldsymbol{B}=0$ and $\boldsymbol{E}=\left(E_{r}, E_{\theta}=0, E_{\phi}=0\right)$, where

$$
E_{r}(r)=\frac{\mathcal{C} \Phi^{-3}(r)}{r^{2}}
$$

and $\mathcal{C}$ is an integration constant. The radial component of the spherically symmetric, Coulomb electric field, resulting from the Maxwell equations, is qualitatively the same [3, Ch. 1]

$$
E_{r}^{M}(r)=\frac{Q(r)}{r^{2}},
$$

where $Q(r)=4 \pi \int_{0}^{r} \rho\left(r^{\prime}\right) r^{\prime 2} d r^{\prime}$ is the electric charge contained by the sphere of radius $r$, centered at the origin and $\rho(\cdot)$ denotes a spherically symmetric, electrical charge density. Comparing Eqs. (5.43) and (5.44) yields $\mathcal{C} \Phi^{-3}(r)=Q(r)$, addressing the integration constant $\mathcal{C}$ and providing an interpretation for $\Phi$, in this restricted geometry. The amended Maxwell equations recover the concept of point-like source electric charge, which is remarkably successful with the model of the hydrogenic atom.

In magnetostatics, we say $\boldsymbol{E}=0$. If $\varrho=\left(\varrho_{\rho}, \varrho_{\phi}=0, \varrho_{z}=0\right)$, in cylindrical coordinates, then the solution of Eq. (5.37) is $\boldsymbol{B}=\left(B_{\rho}=0, B_{\phi}, B_{z}=0\right)$, where

$$
B_{\phi}(\rho)=\frac{\mathcal{C} \Phi^{-3}(\rho)}{\rho}
$$

is reminiscent of Ampère's law. The solution predicts nonzero magnetic field in the absence of ohmic current. In fact, taking a curl on both hand sides of Eq. (5.37) yields a generalized Helmholtz equation

$$
\nabla^{2} \boldsymbol{B}=(\nabla \varrho) \boldsymbol{B} .
$$

We assume that $\nabla \varrho$ is zero outside a compact domain and say that the magnetic sources, $\varrho \times \boldsymbol{B}$, are localized. Thus, Eq. (5.46) becomes the Laplace equation outside the compact domain, so we can choose magnetostatic solutions vanishing at infinity; localized magnetic sources yield localized magnetostatic fields. Traditionally, we acknowledge only ohmic current and spin magnetic moments as the sources of magnetic field. However, our $5 \mathrm{D}$ theory suggests that sources of magnetic field can also be represented as $\boldsymbol{\varrho} \times \boldsymbol{B}$. Given suitable models for $\varrho$, Eq. (5.37) may serve as a basic tool to model field configurations due to magnetic materials [5]. It is very important to note that, the field equations of $4 \mathrm{D}$ mechanics (4.12)-(4.14), and, implicitly, Eq. (5.37), cannot describe the thermodynamics of the electromagnetic field response, addressing topics such as the Curie temperature, the Curie-Weiss law, etc. According to the principles of our 5D theory, this requires the translation along $x^{0}$ be formalism isometry, and a statistic interpretation of the electromagnetic field.

\subsubsection{A new class of field-static problems}

In contrast to the traditional electrostatic and magnetostatic equations, (5.34)-(5.37) allow for another class of problems, besides that of localized field configurations. We consider problems of field configurations, where static, constant field is applied from spatial infinity; n.b., the applied field has zero curl and divergence. These problems cannot be formulated using the Maxwell equations (1.1)-(1.4), where sources are considered to be independent of field, and applying field from spatial infinity has no consequence for the localized field component. In electrostatics, we write $\boldsymbol{E}=\boldsymbol{E}^{(0)}+\boldsymbol{E}^{(1)}$, where $\boldsymbol{E}^{(0)}$ is the field at infinity and $\boldsymbol{E}^{(1)}$ is localized field. Using Eqs. (5.34) and (5.36), and $\boldsymbol{E}^{(1)} \equiv-\nabla A_{0}^{(1)}$, we get

$$
\nabla^{2} A_{0}^{(1)}=\frac{4 \pi}{c} \varrho \nabla A_{0}^{(1)}-\frac{4 \pi}{c} \varrho \boldsymbol{E}^{(0)},
$$

a non-homogenerous equation in $A_{0}^{(1)}$, to be solved using Green's function, starting from the homogeneous equation (5.42). In magnetostatics, we write $\boldsymbol{B}=\boldsymbol{B}^{(0)}+\boldsymbol{B}^{(1)}$, where $\boldsymbol{B}^{(0)}$ is the field at infinity and $\boldsymbol{B}^{(1)}$ is a 
localized. Introducing $\boldsymbol{B}^{(1)} \equiv \nabla \times \boldsymbol{A}^{(1)}$, under the gauge condition $\nabla \boldsymbol{A}^{(1)}=0$, we obtain a non-homogeneous equation in $\boldsymbol{A}^{(1)}$,

$$
-\nabla^{2} \boldsymbol{A}^{(1)}=\frac{4 \pi}{c} \boldsymbol{\varrho} \times\left(\nabla \times \boldsymbol{A}^{(1)}\right)+\frac{4 \pi}{c} \boldsymbol{\varrho} \times \boldsymbol{B}^{(0)},
$$

which, again, can be approached through the method of Green's function. The RHS of Eq. (5.48) has two terms: $\varrho \times\left(\nabla \times \boldsymbol{A}^{(1)}\right)$ represents a current density due to the localized magnetic field $\boldsymbol{B}^{(1)}$, and $\varrho \times \boldsymbol{B}^{(0)}$ represents the contribution to magnetic sources by the magnetic field applied from spatial infinity. Hopes are that Eq. (5.48) can serve to model penetration of magnetic field inside materials and permanent currents. For $\boldsymbol{B}^{(0)}=0$, we choose the solution $\boldsymbol{B}^{(1)}=0$, while for $\boldsymbol{B}^{(0)} \neq 0$, we solve Eq. (5.48) to obtain $\boldsymbol{B}^{(1)}$.

\subsubsection{Another 5D space-time geometry for electrostatics and magnetostatics}

Field configurations can appear static to the 4D observer, even though the 5D geometry does not have a Killing field along $x^{0}$. In particular, the gravitational (i.e., $\tilde{g}_{\mu \nu}$ ) and electromagnetic fields can be observed as being static through observing passive particle propagation. However, time dependence can occur in the lapse field $\Phi$, which is a dark field. The amended Maxwell equations admit time-independent solutions, if $\varsigma$ and $\varrho$ are time-independent. Equation (5.26) further implies that $\varsigma$ must have zero gradient, thus the conductivity $\varsigma$ is a constant field, but $\varrho$ remains a zero-curl 3D field. ${ }^{20}$ The field equations become

$$
\begin{aligned}
\nabla \boldsymbol{E} & =\frac{4 \pi}{c} \varrho \boldsymbol{E}, \\
\nabla \boldsymbol{B} & =0 \\
\nabla \times \boldsymbol{E} & =0 \\
\nabla \times \boldsymbol{B} & =\frac{4 \pi}{c} \varsigma \boldsymbol{E}+\frac{4 \pi}{c} \boldsymbol{\varrho} \times \boldsymbol{B},
\end{aligned}
$$

accommodating better the traditional magnetostatics [3, Ch. 5], which results assuming a perfect conductor; i.e., $\varrho=0$. However, because translation along $x^{0}$ is not a $5 \mathrm{D}$ isometry, our $5 \mathrm{D}$ framework implies that the statistics of the electromagnetic field is ill-defined. In fact, experiment shows that, as long as conductivity is nonzero, the electromagnetic field continuously loses energy through ohmic heating. It may thus be intuitive that, in this case, the electromagnetic field cannot be in thermodynamic equilibrium, even though it is time-independent.

We comment on the original Hall effect, using Eqs. (5.49)-(5.52) to sketch a theory for the timeindependent phenomenon, even though $\varsigma$ and $\varrho$ provide just a crude model for the material properties. In particular, constant conductivity over the entire 3D space necessarily implies that the conducting material fills up the entire 3D space. We assume that the magnetic field has two components, $\boldsymbol{B}=\boldsymbol{B}^{(0)}+\boldsymbol{B}^{(1)}$, where $\boldsymbol{B}^{(0)}$ is a constant magnetic field, applied from infinity, and $\boldsymbol{B}^{(1)}$ is the localized magnetic field. Equation (5.52) yields

$$
-\nabla^{2} \boldsymbol{A}^{(1)}=\frac{4 \pi}{c} \varsigma \boldsymbol{E}+\frac{4 \pi}{c} \boldsymbol{\varrho} \times\left(\nabla \times \boldsymbol{A}^{(1)}\right)+\frac{4 \pi}{c} \boldsymbol{\varrho} \times \boldsymbol{B}^{(0)},
$$

with the same notation as in Eq. (5.48). The electric field $\boldsymbol{E}$ is an input in Eq. (5.53), and results from Eq. (5.47), where we also distinguish two components for the electric field $\boldsymbol{E}=\boldsymbol{E}^{(0)}+\boldsymbol{E}^{(1)}$. The typical conceptualization of the field configuration for the Hall effect $[27,28]$ considers $\boldsymbol{B}^{(0)}$ pointing along the $z$-axis and $\boldsymbol{E}^{(0)}$ pointing along the $x$-axis. Equation (5.53) predicts the existence of a non-ohmic current component due to the magnetic field at infinity, $\varrho \times \boldsymbol{B}^{(0)}$, next to a contribution from $\varrho \times\left(\nabla \times \boldsymbol{A}^{(1)}\right)$, which depends implicitly on $\boldsymbol{B}^{(0)}$ and $\boldsymbol{E}$. These additional current components could be translated into electric field components using Ohm's law, within the perfect conductor model. Still, this is very different from the position of the traditional theory of the Hall effect [27,28], that the electric field $\boldsymbol{E}$ changes with $\boldsymbol{B}^{(0)}$. The Hall coefficient $R_{H}=E_{y} /\left(j_{x} B_{z}\right)$ quantifies this effect and is regarded as a measure of electron density within the conductor material. These developments, based on conduction mechanisms and the perfect conductor model,

\footnotetext{
${ }^{20}$ The field $\ln \Phi^{3}$ depends linearly with time.
} 
are incompatible to our theory. It is very important to note that the experimental measurements, themselves, provide readings of electric current and potential, based on Ohm's law and the perfect conductor model, as well. Therefore, as anticipated in Sec. 5, giving up the model of the perfect conductor, poses problems not only for theory, but also for measurement principles and data interpretation. Our field theoretic approach would require measurement principles based on the principles of general relativity; i.e., field probes using passive particle propagation, rather than measurements of electrical current and potential. Corroborating Eq. (5.53) with experimental results, remains a topic for further work.

\section{Discussion and conclusions}

The electromagnetic theory and experiment developed in parallel during the nineteenth century, both sides contributing to sharpening much needed physical concepts such as field, perfect conductor, and electron. Meanwhile, condensed matter physics also made considerable progress, particularly through combining elements of thermodynamics, electromagnetism and atomic theory. We submit that, owing to the choice of fundamental concepts in electromagnetism, some actual field phenomena ${ }^{21}$ might have been misclassified for describing properties of matter. In consequence, traditional electromagnetism seems very accessible and successful, wherever applicable by itself, whereas condensed matter physics struggles with describing complex properties for matter. A revision of the fundamental concepts in electromagnetism and condensed matter physics may be in order, involving both theory and experiment. Kaluza's proposal, that electromagnetism and gravity should be unified within the 5D general relativity framework, has inspired very much work on the theoretical fundamentals of electromagnetism and gravity. On the experimental side, a very broad set of measurement techniques became available, and may be critically evaluated from the fundamental point of view, taking into account philosophical choices in electromagnetism and general relativity.

In this work, we analyzed the equations of $5 \mathrm{D}$ gravity from the perspective of the Maxwell equations and Ohm's law. The covariance of the 5D metric field can be broken to distinguish 4D gravitational field, electromagnetic field and a dark scalar field. Among the field equations resulting from the 5D condition of Ricci flatness, we recognized Maxwell-type equations and a generalization of Ohm's law, modeling an imperfect conductor, which holds electric charge in volume and can be penetrated by an exterior magnetic field. Furthermore, our analysis makes it clear that the density of electrical charge and current should not be considered as fundamental concepts in electromagnetism. Still, the notion of electrical charge retains fundamental status. Indeed, passive specific charge occurs as a factor converting the electromagnetic field into 5D gravitational field; see Eq. (3.10). Active charge may occur as a parameter of the 5D metric. For example, black hole metrics have parameters such as mass, angular momenta, etc., often called conserved charges. Active electric charge may be found among these parameters.

Based on the 5D gravity equations, we proposed an amendment to the Maxwell equations, in the limit of weak fields, and commented on a few, direct applications. Notably, we discussed electromagnetic wave propagation through an imperfect conductor, electrostatics, magnetostatics of magnetic materials, permanent currents and the Hall effect. The goal of this manuscript is to convey that, even though the electromagnetic theory resulting from $5 \mathrm{D}$ gravity differs in many regards from the traditional theory, it is able to recover much of the formalism, albeit on a slightly different conceptual basis. We did not provide a mathematical definition for the limit of weak fields, rather, we capitalized on the resemblance between the Maxwell equations and 5D gravity equations. There are three main reasons why our discussion of physical applications is limited. First, the 5D space-time may not be Ricci-flat, and a stress-energy tensor representing 5D active matter may be postulated. Second, the $x^{5}$-translational symmetry of the 5D space-time may be only approximate, and the field formalism could be adjusted using perturbation methods to account for the symmetry breaking. Third, the final word is left to exact solutions of the field equations. Therefore, the physics of electromagnetism resulting from $5 \mathrm{D}$ gravity could be significantly more complex.

In conclusion, the geometry of an empty 5D space-time with a space-like Killing field can serve to reconsider the fundamentals of electromagnetism and condensed matter theory. If the 5D space-time has a time-like Killing field, then it may be possible to construct a field thermodynamics. This remains a topic for further work.

\footnotetext{
${ }^{21}$ Hall regarded his discovery as a field effect, going against the Maxwell's conceptualization of electromagnetism [42].
} 


\section{A Alternate forms for the electrostatic potential equation}

Equation (5.42), describing the electrostatic field versus $\varrho$, is not found among the popular partial differential equations of mathematical physics. However, the equation can be reformulated to resemble more popular equations. For example, in the field variable $\alpha \equiv A_{0} \Phi^{3 / 2}$, Eq. (5.42) becomes a generalized Helmholtz equation

$$
\nabla^{2} \alpha-\left[\frac{3}{2} \frac{\nabla^{2} \Phi}{\Phi}+\frac{3}{4} \frac{(\nabla \Phi)^{2}}{\Phi^{2}}\right] \alpha=0 .
$$

Evidently, if $A_{0}$ vanishes at infinity, then so does $\alpha$. The generalized Helmholtz equation has been previously studied, in both two and three dimensions $[37,57,58]$. However, general solutions for the $3 \mathrm{D}$ case are yet to be formulated.

Another possibility is to transform Eq. (5.42) into an integral equation, using the Green's function of the Laplace operator. We obtain

$$
A_{0}(\boldsymbol{x})=-\int d^{3} x^{\prime} \frac{\boldsymbol{\varrho}\left(\boldsymbol{x}^{\prime}\right) \nabla A_{0}\left(\boldsymbol{x}^{\prime}\right)}{c\left|\boldsymbol{x}-\boldsymbol{x}^{\prime}\right|},
$$

which, under the assumption that $\nabla A_{0}$ is a localized field, can be integrated by parts to give

$$
A_{0}(\boldsymbol{x})=\int d^{3} x^{\prime} A_{0}\left(\boldsymbol{x}^{\prime}\right) K\left(\boldsymbol{x}, \boldsymbol{x}^{\prime}\right),
$$

where

$$
K\left(\boldsymbol{x}, \boldsymbol{x}^{\prime}\right)=\frac{\nabla \varrho\left(\boldsymbol{x}^{\prime}\right)}{c\left|\boldsymbol{x}-\boldsymbol{x}^{\prime}\right|}+\frac{\varrho\left(\boldsymbol{x}^{\prime}\right)\left(\boldsymbol{x}-\boldsymbol{x}^{\prime}\right)}{c\left|\boldsymbol{x}-\boldsymbol{x}^{\prime}\right|^{3}} .
$$

Therefore, $A_{0}$ appears as a fixed point (i.e., eigenvector of unit eigenvalue) of the integral operator with kernel $K(\cdot, \cdot)$. Fixed points of integral operators have been previously studied [59-62]. 


\section{References}

[1] E. T. Whittaker. A history of the theories of aether and electricity: From the age of Descartes to the close of the nineteenth century. Longmans and Green, London, 1910.

[2] M. Born and E. Wolf. Principles of optics. Cambridge University Press, 7 edition, 1999.

[3] J. D. Jackson. Classical Electrodynamics. John Wiley \& Sons, Inc, first edition, 1962.

[4] M. Tran. Evidence for Maxwell's equations, fields, force laws and alternative theories of classical electrodynamics. European Journal of Physics, 39(6):063001-31, 2018.

[5] H. Kronmüller and S. S. P. Parkin. Handbook of magnetism and advanced magnetic materials. John Wiley \& Sons, 2007.

[6] T. Kaluza. Zum Unitätsproblem der Physik. Sitzungsberichte der Königlich Preußischen Akademie der Wissenschaften Berlin, pages 966-972, 1921.

[7] P. S. Wesson. Space-time-matter. World Scientific, 1999.

[8] O. Klein. Quantentheorie und fünfdimensionale Relativitätstheorie. Zeitschrift für Physik, 37(12):895906, 1926.

[9] Y. Thiry. The equations of Kaluza's unified theory. Comptes Rendus Hebdomadaires des Séances de l' Académie des Sciences, 226(3):216-218, 1948.

[10] J. M. Overduin and P. S. Wesson. Kaluza-Klein gravity. Physics Reports, 283(5-6):303-378, 1997.

[11] G. Lessner. Unified field-theory on the basis of the projective theory of relativity. Physical Review D, 25(12):3202-3217, 1982.

[12] L. A. López, N. Bretón, and B. V. Ramírez. Einstein-Rosen solutions from Kaluza-Klein theory. General Relativity and Gravitation, 45(1):27-39, 2012.

[13] R. Breban. The 4D Dirac equation in five dimensions. Annalen der Physik, 530(10):1800042-9, 2018.

[14] R. Breban. A five-dimensional perspective on the Klein-Gordon equation. Annals of Physics, 356:158$170,2015$.

[15] R. Breban. Interpretation of the five dimensional quantum propagation of a spinless massless particle. Progress of Theoretical Physics, 114(3):643-668, 2005.

[16] R. Breban. On spinless null propagation in five-dimensional space-times with approximate space-like Killing symmetry. The European Physical Journal C, 76(9):486-4, 2017.

[17] R. Arnowitt, S. Deser, and C. W. Misner. Republication of: The dynamics of general relativity. General Relativity and Gravitation, 40(9):1997-2027, 2008.

[18] C. W. Misner, K. S. Thorne, and J. A. Wheeler. Gravitation. W. H. Freeman, 1973.

[19] R. M. Wald. General Relativity. University of Chicago Press, 2010.

[20] R. E. Reall and Harvey S. Black Holes in Higher Dimensions. Living Reviews in Relativity, 11:6, 2008.

[21] V. P. Frolov, P. Krtouš, and D. Kubiznák. Black holes, hidden symmetries, and complete integrability. Living Reviews in Relativity, 20(1), 2017.

[22] R. Emparan and H. S. Reall. A rotating black ring solution in five dimensions. Physical Review Letters, 88(10):101101, 2002.

[23] G. Randers. On an asymmetrical metric in the four-space of general relativity. Physical Review, 59(2):195-199, 1941. 
[24] X. Cheng and Z. Shen. Finsler geometry: an approach via Randers spaces. Springer, 2012.

[25] L. Roszkowski, E. M. Sessolo, and S. Trojanowski. Wimp dark matter candidates and searches - current status and future prospects. Reports on Progress in Physics, 81(6):066201, 2018.

[26] G. Bertone and D. Hooper. History of dark matter. Reviews of Modern Physics, 90(4):045002, 2018.

[27] N. W. Ashcroft and N. D. Mermin. Solid State Physics. Cengage Learning, 1976.

[28] C. Kittel. Introduction to Solid State Physics. Wiley, 2005.

[29] P. M. Bellan. Fundamentals of Plasma Physics. Cambridge University Press, 2008.

[30] J. A. Bittencourt. Fundamentals of Plasma Physics. Springer Science \& Business Media, 2013.

[31] M. Henry and A. Kassiba. Mini Manuel d'Electromagnétisme. Dunod, 2013.

[32] A.-M. Ampére. Mémoire sur l'action mutuelle entre deux courants électriques, un courant électrique et un aimant ou le globe terrestre, et entre deux aimants. Annales de Chimie et de Physique, 15:59-75 $170-218,1820$.

[33] H. Iguchi, K. Izumi, and T. Mishima. Exact solutions II: Systematic solution-generation of fivedimensional black holes. Progress of Theoretical Physics Supplement, 189(189):93-125, 2011.

[34] S. Tomizawa and M. Nozawa. Vacuum solutions of five dimensional Einstein equations generated by inverse scattering method. II. Production of the black ring solution. Physical Review D, 73(12):636-10, 2006.

[35] A. Billyard and P. S. Wesson. Class of exact solutions in 5D gravity and its physical properties. Physical Review D, 53(2):731-737, 1996.

[36] G. Dotti, J. Oliva, and R. Troncoso. Exact solutions for the Einstein-Gauss-Bonnet theory in five dimensions: Black holes, wormholes, and spacetime horns. Physical Review D, 76(6):064038, 2007.

[37] C. Riveros and A. Corro. Generalized Helmholtz equation. Selecciones Matemáticas, 6(1):19-25, 2019.

[38] S. Alexeyev, N. Popov, M. Startseva, A. Barrau, and J. Grain. Kerr-Gauss-Bonnet black holes: Exact analytical solution. Journal of Experimental and Theoretical Physics, 106(4):709-713, 2008.

[39] A. Anabalón, N. Deruelle, Y. Morisawa, J. Oliva, M. Sasaki, D. Tempo, and R. Troncoso. Kerr-Schild ansatz in Einstein-Gauss-Bonnet gravity: an exact vacuum solution in five dimensions. Classical and Quantum Gravity, 26(6):065002-12, 2009.

[40] L. A. López, O. Pedraza, and V. E. Ceron. Time-dependent solution from Myers-Perry. Canadian Journal of Physics, 94(2):177-179, 2016.

[41] N. Bretón, A. Feinstein, and L. A. López. 5D gravitational waves from complexified black rings. Journal of High Energy Physics, 2010(2):7-20, 2010.

[42] E. H. Hall. On a new action of the magnet on electric currents. American Journal of Mathematics, $2(3): 287-292,1879$.

[43] P. Kapitza. The change of electrical conductivity in strong magnetic fields. Part I. Experimental results. Proceedings of the Royal Society of London. Series A, 123(791):292-341, 1929.

[44] P. Kapitza. The change of electrical conductivity in strong magnetic fields. Part II. The analysis and the interpretation of the experimental results. Proceedings of the Royal Society of London. Series A, 123(791):342-372, 1929.

[45] P. Kapitza. The study of the specific resistance of bismuth crystals and its change in strong magnetic fields and some allied problems. Proceedings of the Royal Society of London. Series A, 119(782):358-443, 1928. 
[46] I. A. Kokurin. Electronic states and persistent currents in nanowire quantum ring. Semiconductors, $52(4): 535-538,2018$.

[47] R. Gréget, G. L. Nealon, B. Vileno, P. Turek, C. Mény, F. Ott, A. Derory, E. Voirin, E. Rivière, A. Rogalev, F. Wilhelm, L. Joly, W. Knafo, G. Ballon, E. Terazzi, J.-P. Kappler, B. Donnio, and J.-L. Gallani. Magnetic properties of gold nanoparticles: A room-temperature quantum effect. ChemPhysChem, 13(13):3092-3097, 2012.

[48] A. C. Bleszynski-Jayich, W. E. Shanks, B. Peaudecerf, E. Ginossar, F. von Oppen, L. Glazman, and J. G. E. Harris. Persistent currents in normal metal rings. Science, 326(5950):272-275, 2009.

[49] D. J. Griffiths. Introduction to Electrodynamics. Prentice Hall, Inc., 1999.

[50] M. Born and E. Wolf. Principles of Optics. Cambridge University Press, 2000.

[51] Z. Jalali-Mola and S. A. Jafari. Electrodynamics of tilted Dirac and Weyl materials: A unique platform for unusual surface plasmon polaritons. Physical Review B, 100(20):205413, 2019.

[52] V. L. Ginzburg. Electromagnetic waves in isotropic and crystalline media characterized by dielectric permittivity with spatial dispersion. Soviet Physics JETP, 34(7):1593-1604, 1958.

[53] G. F. Brand. Enhanced electromagnetic wave transmission through narrow conducting channels. American Journal of Physics, 74(4):289-294, 2006.

[54] J. M. Carcione and F. Cavallini. Forbidden directions for TM waves in anisotropic conducting media. IEEE Transactions on Antennas and Propagation, 45(1):133-139, 1997.

[55] M. A. Baqir and P. K. Choudhury. Investigation of uniaxial anisotropic chiral metamaterial waveguide with perfect electromagnetic conductor loading. Optik, 126(11-12):1228-1232, 2015.

[56] V. L. Farko, S. I. Khankina, and V. M. Yakovenko. Propagation of electromagnetic waves in a conducting magnetic medium. Radiophysics and Quantum Electronics, 43(1):34-40, 2000.

[57] G. Osnabrugge, S. Leedumrongwatthanakun, and I. M. Vellekoop. A convergent Born series for solving the inhomogeneous Helmholtz equation in arbitrarily large media. Journal of Computational Physics, 322:113-124, 2016.

[58] R. P. Shaw and G. D. Manolis. A generalized Helmholtz equation fundamental solution using a conformal mapping and dependent variable transformation. Engineering Analysis with Boundary Elements, 24(2):177-188, 2000.

[59] R. Datko. A fixed-point theorem for a class of integral operators. Journal of Mathematical Analysis and Applications, 23(3):704-713, 1968.

[60] M. U. Ali, P. Kumam, and F. Fahimuddin. Existence of fixed points for an integral operator via fixed point theorem on gauge spaces. Communications in Mathematics and Applications, 9(1):15-25, 2018.

[61] M. Nazam and M. Arshad. On a fixed point theorem with application to integral equations. International Journal of Analysis, 2016(1-2):1-7, 2016.

[62] R. V. Rao and N. Sukavanam. On the eigenvalues and eigenfunctions of some integral operators. Journal of Mathematical Analysis and Applications, 109(2):463-471, 1985. 